\title{
His Bundle Pacing: Short Review of Literature and Technical Aspects
}

\section{Mukherjee $S^{*}$}

Institute of Cardiovascular Science, Institute of Post Graduate Medical Education and Research, Kolkata, India

${ }^{*}$ Corresponding author: Mukherjee S, MBBS, MD (Medicine), DM (Cardiology)-Post Doctorate Fellow, Institute of Cardiovascular Science, Institute Of Post Graduate Medical Education and Research, Kolkata 700020, India, Tel: 9163097595, E-mail: drsumukherjee@gmail.com

Citation: Mukherjee S (2018) His Bundle Pacing: Short Review of Literature and Technical Aspects. J Clin Exp Res Cardiol 4(2): 201

Received Date: August 11, 2018 Accepted Date: October 16, 2018 Published Date: October 18, 2018

\begin{abstract}
History of pacing has evolved from the primitive state of temporary transvenous pacing by large fixed machine to leadless modern technology. The pacing site has also been changed from traditional right ventricular apex to various other sites. The concept behind such experient was due to several complications in the long term from traditional Right Ventricular Apical Pacing (RVAP). The His Bundle pacing results in conduction and contraction similar to and and more often called physiological pacing. This mini review describes short history of evolution of concept of His Bundle pacing and its technical aspects of successful implantation.
\end{abstract}

Keywords: Right Ventricular Apical Pacing (RVAP); Physiological Pacing; His Bundle pacing (HBP)

\section{RV Apical Pacing and the Consequences}

From the first human implantation the right ventricular (RV) apical pacing has saved millions of lives, but within one decade it was proved to be non-physiological as causes several deleterious hemodynamic effects. Right ventricular apical pacing alters left ventricular electrical and mechanical activation. Chronic right ventricular apical pacing causes left ventricular dilatation and reduction of left ventricular ejection fraction [1]. During RV apical pacing, the conduction of the electrical wave front propagates through the myocardium, rather than through the His-Purkinje conduction system. As a result the electrical wave form propagates more slowly and induces heterogeneity in electrical activation of myocardium comparable to left bundle branch block [2]. RVAP is associated with a diminished rate of change in left ventricular pressure (dp/dt) and an abnormal dyssynchronous contraction pattern [3]. RV apex has been shown to decrease the contractile state of normal myocardium due to abnormal electrical propagation. Not only functional but anatomical changes have also been demonstrated in several previous studies that includes various histopathologic abnormalities in paced patients. Histopathological alter $\neg$ ations in biopsy samples following pacing include myofiber variation, fibrosis, fat deposition, sclerosis, and mito $\neg$ chondrial morphological changes [4]. These findings indicate that chronic RV apical pacing may adversely alter myocellular growth at the cellular and subcellular levels. Unfavorable effects of right ventricular (RV) pacing include ventricular remodeling, dilation, elevated diastolic filling pressure, increased functional mitral regurgi $\neg$ tation, myocardial perfusion defects, and reduced LV ejection fraction. Of all ventricular sites, the RV apex seems to be the most hemodynamically unfavorable [5]. Right ventricular apical pacing (RVAP) has been shown to increase morbidity and mortality in patients receiving a high percentage of cumulative pacing [6]. All these factors lead to search for alternative pacing site. Various alternative pacing sites has been explored that includes Right Ventricular Outflow Tract pacing, Septal Pacing, Biventricular Pacing, Atrial Pacing, Dual Pacing and His Bundle pacing.

\section{Evolution of His Bundle Pacing}

The term 'physiological' was used in Canadian Trial of Physiological pacing (CTOPP) to reflect the terminology at the time of development of the trial [7]. However atrial pacing was considered physiological at that time. Later it was thought that pacing the atrium and ventricle sequentially may solve the problem of unsynchronised contraction and prevent atrial bradycardia but the ventricular activation sequence is clearly not physiological. Studies using biventricular pacing have suggested improvement in patients of left bundle branch block. The dual chamber and VVI implantable defibrillator (DAVID study) randomised patients receiving implantable defibrillators (ICD) either to backup pacing at the rate of $40 \mathrm{bpm}$ or to DDDR pacing at the at the rate of 70 per minutes. The composite end point of death or hospitalisation with heart failure was greater in the group receiving DDDR pacing than in the back up pacing group [8]. There was a very similar benefit with regard to reduction of atrial fibrillation and a significant but weak benefit with regard to hospitalisation for heart failure. Two probable solutions to this problem are: first involves manipulation of pacing modes and timing cycle operation among patients with reliable atrio-ventricular (AV) 
conduction to minimise unnecessary ventricular pacing and preserve normal ventricular conduction and the second involves pacing at alternative ventricular site to attenuate the adverse effects imposed by ventricular desynchronization when ventricular pacing can't be avoided.

\section{What is Physiological Pacing?}

The main purpose of permanent cardiac electrostimulation is to maintain an adequate cardiac rhythm, trying to restore the physiology of the normal excito-conductive physiology of the heart as much as possible. Up until now, importance had been given to two elements that were considered fundamental for physiological pacing: maintenance of the atrioventricular Sequence and the rate-responsive function. Pacemakers, therefore, were considered "physiological".

If the aim is to mimic physiological activation patterns, the HB undoubtedly provides the ideal site. The first set of data comes from a team in Pennsylvania showed that Long-term, directs his pacing results in a reduction of left ventricular dimensions and improved cardiac function in patients with atrial fibrillation and dilated cardiomyopathy. During the last several years there was much work done in the field of DHBP, to extend the indications and to improve the technique.

\section{Technical details of successful His Bundle Pacing}

\section{HB localization}

$\mathrm{HB}$ recording was obtained using a quadripolar catheter with 2-5 mm electrode spacing introduced from the left femoral vein and advanced to the right atrium under fluoroscopy, the catheter was positioned in the anatomical position of the HB in the superior septal aspect of the TV, by advancing the catheter to the RV in right anterior oblique (RAO) projection and applying a clock wise torque and confirming the septal position in left anterior oblique (LAO) projection. Recording from the catheter is displayed on the cardiolab EP recording system the lead is then screwed in this position by means of $4-5$ clockwise rotations. The HB capture threshold is assessed and accepted if found to be $2.5 \mathrm{~V}$ at $1.0 \mathrm{~ms}$

\section{Procedure}

HBP is performed using the pacing lead delivered through a fixed curve sheath. The delivery sheath initially inserted into the right ventricle beyond the tricuspid annulus over a guide wire through left/right cephalic or axillary vein. Subsequently, the pacing lead is advanced through the sheath such that only the distal electrode /screw are beyond the tip of the catheter. A unipolar electrogram was recorded from the lead tip at again setting of $0.05 \mathrm{mV} / \mathrm{mm}$ and displayed on a monitor of pacing system. The preformed double curve of this catheter points the tip toward the superior AV septum. An HB electrogram is identified by mapping the AV septum. If HB recording is obtained pacing was started to capture the heart, 12 lead ECG simultaneously recorded should show a QRS width less than $120 \mathrm{~ms}$, or the output was reduced until loss of capture or QRS width reduced. Once stable pacing is obtained the pacing threshold is determined, with $2.5 \mathrm{~V}$ or less at $50 \mathrm{~ms}$ is considered acceptable. A screw-in active fixation lead is used for active fixation. The lead was manipulated to aim at the recording tip of the quadripolar catheter. Once acceptable the screw was protruded to fix the lead in position. The pacemaker is programed to sense the patients QRS and not the P wave-if any- by adjusting the sensing threshold.

\section{Review of Literature}

First observation regarding HB electrophysiological characteristics was perhaps done by Scherlag in 1967 [9]. Narula in 1977 reported that, , stimulation from the proximal HB showed wide QRS complexes and/or axis deviation identical to that seen during normal sinus rhythm, whereas distal HB stimulation resulted in a narrow QRS complex (95 ms) and/ or abolition of left axis deviation (LAD) [10].

In 1979 El Sherif underlined how temporary HB stimulation can revert a pre-existing bundle branch block both in canine experimental model and in seven patients with broad QRS after myocardial infarction who underwent temporary His bundle stimulation during permanent Pace Maker implantation procedure [11].

Mabo et al. were the first who achieved a permanent recording and pacing of the His bundle. In 13 anesthetized dogs HB electrogram was retrogradely registered from the aortic root. A deflectable tip multipolar lead was placed under fluoroscopy via the right jugular vein into the right ventricle under the tricuspid septal leaflet in $7 \mathrm{dogs}$; in the other six the same placement was made by palpation through the right atrial wall. Stable His-bundle and right bundle branch recordings were made from distal and proximal electrode pairs, respectively. Consistent His-bundle pacing was accomplished both from the aortic root and from the tricuspid leaflet [12].

In 1999 Amitani confirmed the feasibility of clinical application of permanent HB pacing in six adult beagles in which a screw-in lead was anchored in the His-bundle region [13].

Deshmukh et al. first described successful permanent His-bundle pacing (HBP) in a small series of patients with AF and dilated cardiomyopathy in 2000, which have demonstrated that it is feasible and associated with an improvement in exercise capacity, 
ventricular synchrony, and LVEF as compared with RVP. The success rate of DHBP in this study was 66\% [14].

The success rate of permanent HBP reported in the literature varies significantly from $44 \%$ to $95 \%$, depending on the implantation tool used and the type of patients studied.

Barba-Pichardoetal reported asuccess rate of $65 \%$ in patients with AV block in whom permanent HBP was attempted. This study used standard pacing leads with retractable screws and manually shaped stylets [15].

Zanonetal reported a success rate of $95.5 \%$ in an observational series of 307 patients. This study used HB catheter in all group of patients [16].

Recently Sharma et al. investigated the feasibility, safety and the clinical outcomes of unselected patients who underwent His bundle permanent pacing compared to right ventricular pacing [17].

In 2012 Kronborg et al. demonstrated that HB pacing preserves LV systolic performance and mechanical synchrony as compared with RV apical pacing. Thirty-eight patients with high-grade atrio-ventricular block and narrow QRS complex underwent two conventional bipolar, active fixation pacing leads positioning in the right atrial appendage and on the RV septum, respectively. A third lead was positioned on HB level after mapping of the Hisian area with a diagnostic catheter [18].

The long term results of Kronborg et al. published in 2013 analyzed data from 38 patients that underwent HB pacing and RV septal pacing in a 12 month crossover study. His or para-His pacing preserves $\operatorname{LVEF}(55 \%$ vs $50 \%, \mathrm{p}=0.005)$ and mechanical synchrony compared with RV septal pacing in patients with a high-grade atrio-ventricular block, a narrow QRS width, and LVEF N40\%. In this population of patients, His bundle or para-His pacing might potentially prevent pacing-induced heart failure.

\section{Conclusion}

Physiological pacing 'may be achieved only by preserving, or, if that is impossible, by restoring or attempting to imitate the normal electrophysiological characteristics of the heart. Because His-bundle pacing (HBP) produces ventricular contraction via the specific conduction system, it does not induce interventricular or intraventricular asynchrony or trigger the myocardial perfusion disorders described with right ventricular apical pacing. His-bundle pacing is has been shown to be effective in cases of supraHisian blocks. It can also correct many conduction disturbances usually considered to be infra-Hisian and, therefore, can be used in selected cases under such circumstances.

Although HBP poses little challenge in terms of technical aspects but not very difficult now, thanks to improvements in different long sheaths and various screwing active fixation system. Thorough anatomical knowledge however is very much essential to avoid damage to Atro ventricular node during implantation.

\section{References}

1. Shukla HH, Hellkamp AS, James EA, Flaker G, Lee K, et al. (2005) Heart failure hospitalization is more common in pacemaker patients with sinus node dysfunction and a prolonged paced QRS duration. Heart Rhythm 2: 245-51.

2. Cho GY, Kim MJ, Park JH, Kim HS, Youn HJ, et al. (2011) Comparison of ventricular dyssynchrony according to the position of right ventricular pacing electrode: A multi-center prospective echocardio $\neg$ graphic study. J Cardiovasc Ultrasound 19: 15-9.

3. Karpawich PP, Rabah R, Haas JE (1999) Altered cardiac histology following apical right ventricular pacing in patients with congenital atrioventricular block. Pacing Clin Electrophysiol 22: 1372-7.

4. Lindsay BD (2009) Deleterious effects of right ventricular pacing. N Engl J Med 361: 2183-5.

5. Prinzen FW, Augustijn CH, Arts T, Allessie MA, Reneman RS (1990) Redistribution of myocardial fiber strain and blood flow by asynchronous activation. Am J Physiol 259: H300-8.

6. Connolly SJ, Kerr CR, Gent M, Roberts RS, Yusuf S, et al. (2000) Effects of physiologic pacing versus ventricular pacing on the risk of stroke and death due to cardiovascular causes. Canadian Trial of Physiologic Pacing Investigators. N Engl J Med 342: 1385-91.

7. Wilkoff BL, Cook JR, Epstein AE, Greene HL, Hallstrom AP (2002) Dual-chamber pacing or ventricular backup pacing in patients with an implantable defibrillator: the Dual Chamber and VVI Implantable Defibrillator (DAVID) Trial. JAMA 288: 3115-23.

8. Scherlag BJ, Kosowsky B, Damato A (1967) A technique for ventricular pacing from the His bundle of the intact heart. J Appl Physiol 22: $584-7$.

9. Connolly SJ, Kerr CR, Gent M, Roberts RS, Yusuf S, et al. (2000) Effects of physiologic pacing versus ventricular pacing on the risk of stroke and death due to cardiovascular causes. N Engl J Med 342: 1385-91.

10. Sweeney MO, Hellkamp AS, Ellenbogen KA, Greenspon AJ, Freedman RA, et al. (2003) Adverse effect of ventricular pacing on heart failure and atrial fibrillation among patients with normal baseline QRS duration in a clinical trial of pacemaker therapy for sinus node dysfunction. Circulation 107: 2932-7.

11. Nielsen JC, Kristensen L, Andersen HR, Mortensen PT, Pedersen O L, et al. (2003) A randomized comparison of atrial and dual-chamber pacing in177 consecutive patients with sick sinus syndrome: echocardiographic and clinical outcome. J Am Coll Cardiol 42: 614-23.

12. Fung JW, Yu CM (2010) Should we switch to RVOT pacing for all now? Not yet. J Cardiovasc Electrophysiol 21: 1120-6.

13. Shimony A, Eisenberg MJ, Filion KB, Amit G (2012) Beneficial effects of right ventricular non-apical vs. apical pacing:a systematic review and meta-analysis of randomized-controlled trials. Europace 4: 81-91.

14. Deshmukh P, Casavant D, Romanyshyn M, Anderson K (2000) Permanent direct His bundle pacing: a novel approach to cardiac pacing in patients with normal His- Purkinje activation. Circulation 101: 869-77. 
15. Cerqueira MD, Weissman NJ, Dilsizian V, Jacobs AK, Kaul S, et al. (2002) Standardized myocardial segmentation and nomenclature for tomographic imaging of the heart: a statement for healthcare professionals from the Cardiac Imaging Committee of the Council on Clinical Cardiology of the American Heart Association. Circulation 105: 539-42.

16. Suffoletto MS, Dohi K, Cannesson M, Saba S, Gorcsan J (2006) Novel speckle tracking radial strain from routine black-and-white echocardiographic images to quantify dyssynchrony and predict response to cardiac resynchronisation therapy. Circulation 113: 960-8.

17. Hawkins NM, Petrie MC, MacDonald MR, Hogg KJ, McMurray JJ (2006) Selecting patients for cardiac resynchronization therapy: electrical or mechanical dyssynchrony? Eur Heart J 27: 1270-81.

18. Pitzalis MV, Iacoviello M, Romito R, Massari F, Rizzon B, et al. (2002) Cardiac resynchronization therapy tailored by echocardiographic evaluation of ventricular asynchrony. J Am Coll Cardiol 40: 1615-22. 